\title{
Nurses' Perceptions Of MMR Vaccine Hesitancy In An Area With Low Vaccination Coverage
}

This article was published in the following Dove Press journal:

Pediatric Health, Medicine and Therapeutics

\author{
Asha Jama ${ }^{1,2}$ \\ Ann Lindstrand ${ }^{3}$ \\ Mona $\mathrm{Ali}^{\mathrm{I}}$ \\ Robb Butler ${ }^{4}$ \\ Asli Kulane ${ }^{2}$
}

'The Public Health Agency of Sweden, Stockholm, Sweden; ${ }^{2}$ Equity and Health Policy Research Group, Department of Public Health Sciences, Karolinska Institute, Stockholm, Sweden;

${ }^{3}$ Department of Immunization, Vaccines, and Biologicals, World Health Organization, Geneva, Switzerland; ${ }^{4}$ United Nations Children's Fund Programme Division, UNICEF, New York, NY, USA

Correspondence: Asli Kulane Equity and Health Policy Research Group, Department of Public Health Sciences, Karolinska Institutet, Tomtebodavägen I8A, Stockholm 17177, Sweden

Tel +46 8-52483388

Email Asli.Kulane@ki.se
Background and aim: Measles, mumps, and rubella vaccine (MMR) coverage is low in an area in the Northern part of Stockholm, Sweden. The overall aim of this study was to explore the perceptions, views, and experiences of child health clinic nurses related to vaccine hesitancy in Rinkeby and Tensta.

Methods: The study focused specifically on hesitancy towards measles-mumps-rubella (MMR) vaccination. Eleven in-depth interviews were conducted in Swedish with female nurses who were directly responsible for vaccination programmes. Their thoughts and experiences were sought using probing and question rephrasing techniques. Transcripts of the data were analysed using content analysis.

Results: Four themes emerged, namely hesitancy among Somali parents, lack of confidence in the MMR vaccine, loss of confidence in other vaccines due to mistrust of the MMR vaccine, and complacency regarding vaccination in general.

Conclusion: There is a strong influence of fear of vaccine-induced autism among parents in the Somali community in areas of Stockholm, that requires continued attention by the health care providers.

Keywords: MMR vaccine, measles, vaccine hesitancy, nurses, Stockholm

\section{Background}

Research has demonstrated that vaccination is an effective way to combat infectious diseases and the most cost-effective health intervention available, ${ }^{1}$ however cases of such conditions continue to exist among the under-vaccinated communities. ${ }^{2}$ Vaccine hesitancy has been a contributing factor for under-vaccination in some communities $^{3-5}$ and reasons behind this hesitancy are difficult to ascertain. ${ }^{6,7}$

Sweden's measles-mumps-rubella (MMR) vaccination coverage has been consistently high (around 97\%) over the past two decades. However, this national average masks pockets of low coverage, such as in the Rinkeby and Tensta districts of Stockholm. Child Health Clinics (CHCs) in Rinkeby and Tensta reported MMR coverage of $71.5 \%$ and $69.7 \%$ respectively, and have remained low since the late 1990s when Andrew Wakefield published an article in the Lancet on a presumptuous link between autism and the MMR vaccine. The article was later refuted.

Reasons for vaccine hesitancy include information gaps, vaccine safety, parental mistrust of the healthcare system and influence from social networks. ${ }^{8-13}$ Addressing these challenges requires an understanding of the context and opinions of both healthcare consumers (parents) and providers (nurses) in the under-immunized communities. ${ }^{14-16}$ Health care providers (HCP) have been shown to influence decisions to vaccinate. ${ }^{17,18}$ A large review of parents' views and experiences on 
communication about childhood vaccinations demonstrated that parents use health care providers as an important source of information about vaccines. Not only are HCP an important source for vaccine information but they can impact parental decision-making. The qualitative studies showed that if parents perceived a bad relationship or communication with the HCP it could have a negative impact on their vaccine decision. ${ }^{19}$ Other studies have shown the challenges experienced by providers in dealing with hesitant parents ${ }^{20}$ and these challenges could provide insights for other health workers who are dealing with vaccine hesitancy in their communities. ${ }^{21}$

$\mathrm{CHC}$ nurses play a critical role in vaccination delivery, which takes place at CHCs at the local level, however, there is limited knowledge about their views of hesitant parents. $^{22}$ The nurses are the driving force of the entire programme and they interact directly with parents, who have varying opinions about MMR vaccines. The overall aim of this project was to explore the perceptions, views, and experiences of $\mathrm{CHC}$ nurses related to vaccine hesitancy among parents in an area with low vaccination coverage.

\section{Methods}

\section{Research Design And Setting}

The study design is explorative with inductive qualitative approach. The study was conducted in Rinkeby and Tensta, which are districts located in the northwest part of Stockholm that have a high percentage of residents with foreign backgrounds. The population of Rinkeby was 16,047 in 2013, including 1638 children under five years of age. The population of Tensta was 18,866, including 1673 children under five years of age. An estimated $30 \%$ of the population of both Rinkeby and Tensta is of Somali origin. ${ }^{22}$

The study was conducted at the $\mathrm{CHCs}$ in the two districts. This study followed the principles of the World Medical Association and the Declaration of Helsinki ${ }^{23}$ and was approved by the Regional Ethics Committee (Dnr 2013/678-31/3) in Stockholm Sweden.

\section{Participants}

All nurses at the $\mathrm{CHCs}$ were invited to participate in the interviews, none have declined. Written and verbal information was provided to confirm their voluntary participation and guarantee participants' confidentiality and the option to discontinue participation at any time during the study. The researchers' contact information was also provided. All eleven (11) invited nurses consented to participate and gave informed consent in writing.

\section{Data Collection}

Interviews were conducted by Asha Jama (A.J) and Mona Ali (M.A) at the CHCs and venue according. The interviews were conducted in Swedish and on average lasted for 30 to 60 minutes. Qualitative in-depth interviews (IDI) were an appropriate method of collecting information because they enabled the researchers to obtain detailed information about the participants' thoughts and their description of phenomena under study. ${ }^{24}$ Interviews were guided by a few open-ended questions that prompted the nurses to give uninterrupted answers about the beliefs and attitudes of hesitant parents. The questions included: Can you tell me your experience related to hesitant parents at the child health clinics? Supportive questions were posed to obtain more detailed answers from the nurses. The interviewers used probing and question rephrasing techniques to clarify answers and obtain further details from the participants. All collected data was not identity linked to ensure anonymity and to keep the research group within locked facilities.

\section{Data Analysis}

Content analysis was used to analyse data from this study was considered to be suitable method of analysis in order to reveal variation in the content as narrated by the interviewees. All interviews were audio-recorded and transcribed verbatim in Swedish. ${ }^{25}$ Data analysis was performed by the research team immediately after the data collection. Final analysis was conducted by the researchers to make use of their multidisciplinary views (public health staff, medical doctors, social scientist) read the transcripts of the data and all the transcribed interviews were analysed. ${ }^{25}$ Meaning units and later the condensed meaning units were used to generate codes. The categories of coded information were analysed and four themes emerged as illustrated in the below results section. To ensure accuracy and validity, all of the authors were involved in the data analysis. Relevant quotes were selected to support findings and to demonstrate the experiences of the participants and their perceptions.

\section{Results}

\section{Participants' Characteristics}

The study consisted of 11 female nurses with a range of work experience from 2.5 to 45 years. They were all aged between 35 and 67 years and were directly responsible 
for the vaccination programmes. Most were paediatric nurses, however, district nurses were also interviewed. The major themes that emerged were: vaccine hesitancy among Somali parents, lack of confidence in MMR vaccine, loss of confidence in other vaccines due to mistrust of MMR and complacency regarding vaccination in general.

\section{Vaccine Hesitancy Among Somali Parents}

The nurses reported that in their experience most parents who declined to have their children vaccinated with the MMR vaccine were of Somali origin. The parents would normally have decided not to vaccinate before coming to the $\mathrm{CHC}$, and in most cases were not willing to listen to any advice that could persuade them to vaccinate, regardless of who provided the information:

[...]. Yes, it is the Somali group that is the commonest. It happens that few other people don't want to come for vaccination but yes, the largest group is the Somali. [CHC nurse 1]

Although the nurses identified the hesitant group to be mainly parents with Somali origin in Rinkeby and Tensta, the parents did not share other social-demographic characteristics. The hesitant group included new mothers who had just moved to the area, as well as mothers with many children who had resided in the area for a long time. All of the nurses believed that the women in the community were telling each other to avoid vaccines. The nurses were surprised to note that even mothers with high education were hesitant:

No, I don't know but most people, even those who are highly educated. There is a family with a father who is a doctor and he knows very well that this vaccine is very important, but decided otherwise. He left the responsibility to the mother and she decided that the children should not be vaccinated. [CHC nurse 9]

The nurses stressed that this was a localized problem in only Rinkeby and Tensta and that other districts in Stockholm did not have such resistance to vaccines.

The nurses reported that the Somali community has a large social circle, and religion did not appear to be a driver of vaccine hesitancy.

[...] Then the family is very large. It's not just what you know; it is the mother-in-law, grandmother, cousins, aunties, uncles $[\ldots]$, all sorts of people who are part of the family. Then religion and the Imam, yes, they certainly have a role too, but I don't believe that religion is against vaccines in any way. I do not think they have a negative attitude in any way, I don't think so. [CHC nurse 3]

\section{Lack Of Confidence In MMR}

The nurses reported that this group is specifically hesitant towards the MMR vaccine, which they believe is closely linked to autism. This fear that their children would become autistic after getting vaccinated was reported to be the center of their concern and deeply rooted in the community, as explained below;

[...] hhmm some respond to me that they absolutely believe that they would like to vaccinate but are afraid that their children will become autistic and won't start to talk. And they say that they would never in their wildest imagination give such a vaccine with side effects. They say, "It is not possible you think I could do that!" I try to influence them to think otherwise but they have a very strong idea. So it has to come from within them, I think [...]. [CHC nurse 5]

The nurses reported that they had heard most of the stories related to autism being spread by the women in the community. Peer pressure thus appeared to be a strong deterrent factor for the mothers to accept the MMR vaccination.

[...] Yes, it is autism. That the children cannot speak, yes. I have never got any other explanation [...]. Yes, and it certainly plays a role that many friends and acquaintances have children with autism and they have seen them. Autism is very widespread as you know and it can be that children cannot do anything on their own and they are therefore afraid. [CHC 5 nurse]

The nurses reported both mothers and fathers would politely decline the vaccine saying they would seek consultation from their families; however in some situations, the grandmothers accompanied them during the vaccine visits. These grandmothers actively stopped mothers from accepting the vaccine. The nurses reported that the situation was even more difficult for mothers from families with autistic children. These further spread the myth to the other parents, advising them not to vaccinate their children.

[...] no, but the children do not start talking and we have heard that one can get autism. Families that have autistic children that were vaccinated definitely did not vaccinate their second child. [CHC nurse 4] 


\section{Loss Of Confidence In Other Vaccines Due To MMR}

The $\mathrm{CHC}$ nurses said that they recognized a new trend among Somali parents. Some of the parents had started refusing the third dose of diphtheria, tetanus, pertussis, polio, and Hib (Haemophilus influenzae bacteria type b) vaccine, which is administered when the child reached one year. The parents confused the vaccination provided at the age of one year with MMR, which is scheduled at 18 months of age. This factor led to some parents declining to vaccinate their children for both vaccines. Some nurses reported that some parents were afraid the nurses would proceed with administering the vaccination without telling the parents and give the MMR instead of the vaccination for one-year-old children.

[...] some confuse MMR and the vaccination dose three as we call it for, the one-year-old vaccine and they don't take it either. [CHC nurse 6]

$[\ldots]$ they often confuse it with the third vaccine $[\ldots]$ with MMR so they don't attend when the child is one year old and should have his third vaccine because they think it is MMR. I have had many of those cases and I have tried and sate with them and (explain) this is this and that is that and this is MMR [...] bla bla I tried different possible ways so they understand. [CHC nurse 7]

Sometimes they are afraid that one will trick them and give the MRR vaccine at one year, so sometimes they don't even come at one year. In fear of it being measles, mumps, and rubella. We are not going to vaccinate more because we don't what it is. But it is the same as the three months, are you sure. If you get hold of them, then they may take it. But if they are late with the one-year-old vaccine and they become fifteen months it's better not to come so they don't get the eighteen months vaccine wrongly. [CHC nurse 11]

I have a mother with an autistic child and she believes that I will inject it like trick her; she believes it every time we are going to vaccinate. She vaccinates for the other vaccines but she believes and is very worried over that I may give the vaccine against her will, so there is a suspicion that we forcefully vaccinate people. [CHC nurse 4]

The nurses explained that parents even missed the whole visit in order to avoid the third dose of diphtheria, tetanus, pertussis, polio, and Hib (Haemophilus influenzae type b) vaccine. A couple of the nurses expressed their worries that a new rumor, about this vaccine at one year of age, would be received similar to the one about the MMR vaccine among the Somali parents. The nurses said that parents confused MMR with the other vaccines within the vaccination programme and that it is difficult to explain to the parents that it is not the same vaccine they are afraid of. A nurse said that she met a father who refused to vaccinate his son at five-years of age-, with the fourth dose of diphtheria, tetanus, pertussis, polio and Hib vaccine because he believed it could cause autism. Previously parents delayed or refused the MMR at 18 months, however, nurses could see a spill-over of fear to other vaccines within the vaccination programme.

I had a father here today and his boy is five years old and should get the five year old vaccine ... The mother had booked the visit and then the father came and wondered why we had booked this visit, so I told him that the mother wanted to vaccinate ... No, I don't want the vaccine [...] because he had heard from his friends that the five year old vaccine one stops talking. It became very strange to explain that this vaccine is not the one you get autism of, you don't get autism from any vaccine. But I said like I know many Somalis believe that a vaccine cause (autism) but it is not that one. But it didn't matter he didn't want the vaccine because he heard the rumour even about the five year old vaccine. [CHC nurse 4]

\section{Complacency Towards All Vaccines}

During the course of the interviews, it emerged that some of the parents do not accept any vaccines. Although they were reported to be few, these parents believed that the diseases are not so dangerous. The majority of these parents were actually well informed about how vaccines work, but still not willing to vaccinate.

[...] Yes, it does, but I have a feeling that they are really well studied and very knowledgeable about why they do not want to vaccinate. So I just have some families that do not vaccinate at all. [CHC nurse 11]

\section{Discussion}

Fear of MMR vaccine side effects has been observed in the Somali community residing in Rinkeby and Tensta. This fear is essentially driven by a perceived risk that a child may not learn to speak or may develop autism after vaccination, as suggested in earlier studies. ${ }^{12,26}$ The fear is evident among those who are new in the area and those who have resided in this area for a long period. Peer pressure contributes to spreading the perceived myth about MMR vaccine and this spills over to affect trust in other vaccines. 
Downs et al. have illustrated the vulnerability of these parents to negative anti-vaccination information, and this vulnerability poses a challenge for healthcare providers, ${ }^{27}$ as it may block children's opportunity to get vaccinated. ${ }^{15,28}$ The current study shows the importance of the family and peer group in spreading the autism myth and how grandmothers, in particular, can impact parents' confidence in the vaccine.

The role of healthcare providers in maintaining open communication with the parents and winning their trust has been cited as a very important step in increasing vaccine acceptance. ${ }^{28}$ Our results indicate that hesitant parents in this community tend to mistrust the nurses providing the vaccines, judging by the expressed fear that the nurses would trick them into accepting a vaccine that might harm their children.

The Somali community residing in Rinkeby and Tensta is well established, with strong social ties within and among families.

Providing proper information and tailoring programmes to the local context would be essential to enable a conducive environment for the respective vaccination programmes, while tailoring programs to the local context of those communities. ${ }^{29,30}$ Interventions that have been evaluated in other studies indicate that communication strategies can only be successful if they address the target group's key concerns. ${ }^{31}$ Interventions to combat vaccine hesitancy can therefore only be effective if issues of trust and open communication are addressed. $^{32}$

\section{Methodological Considerations}

To ensure trustworthiness several steps were undertaken. Interviews were conducted in Swedish and recorded to authenticate the quotes. Individual interviews were conducted to permit open discussion and room for more probing for deeper understanding. Triangulation was done by researchers in analysing data and preliminary findings were shared with child health experts to increase the credibility of the data. A member check with the health care providers was also conducted. They re-emphasized that some parents postpone appointments as a polite way of turning down the offer to immunize. Qualitative research studies focus to address specific problems in subgroups within a population or community therefore generalizability is not an expected attribute to show that findings are applicable to other situations or populations.

\section{Conclusion}

This study identifies that there is a strong influence of fear of vaccine-induced autism among parents in the
Somali community in areas of Stockholm, Sweden, that requires continued attention by the health care providers. It is important to build trust in the healthcare system by fostering knowledge and improving communication between the parents and the vaccine providers.

\section{Acknowledgment}

The study was funded by the WHO Regional Office for Europe.

\section{Disclosure}

Dr Ann Lindstrand reports grants from WHO Europe during the conduct of the study, and grants for other projects from WHO Geneva, outside the submitted work. The authors report no other conflicts of interest in this work.

\section{References}

1. Hickler B, Guirguis S, Obregon R. Vaccine special issue on vaccine hesitancy. Vaccine. 2015;33(34):4155-4156. doi:10.1016/j.vaccine.2015. 04.034

2. Gahr P, DeVries AS, Wallace G, et al. An outbreak of measles in an undervaccinated community. Pediatrics. 2014;134(1):e220-228. doi:10.1542/peds.2013-4260

3. Alfredsson R, Svensson E, Trollfors B, Borres MP. Why do parents hesitate to vaccinate their children against measles, mumps and rubella? Acta Paediatr. 2004;93(9):1232-1237. doi:10.1111/j.1651-2227.2004. tb02755.x

4. Kestenbaum LA, Feemster KA. Identifying and addressing vaccine hesitancy. Pediatr Ann. 2015;44(4):e71-75. doi:10.3928/0090448120150410-07

5. MacDonald NE, Hesitancy SWGoV. Vaccine hesitancy: definition, scope and determinants. Vaccine. 2015;33(34):4161-4164. doi:10.1016/j. vaccine.2015.04.036

6. Schuster M, Eskola J, Duclos P, Hesitancy SWGoV. Review of vaccine hesitancy: rationale, remit and methods. Vaccine. 2015;33 (34):4157-4160. doi:10.1016/j.vaccine.2015.04.035

7. Larson H, Jarrett C, Eckersberger E, Smith D, Paterson P. Understanding vaccine hesitancy around vaccines and vaccination from a global perspective: a systematic review of published literature, 2007-2012. Vaccine. 2014;32(19):2150-2159. doi:10.1016/j.vaccine. 2014.01.081

8. Smailbegovic MS, Laing GJ, Bedford H. Why do parents decide against immunization? The effect of health beliefs and health professionals. Child Care Health Dev. 2003;29(4):303-311. doi:10.1046/ j.1365-2214.2003.00347.x

9. Brown KF, Kroll JS, Hudson MJ, et al. Factors underlying parental decisions about combination childhood vaccinations including MMR: a systematic review. Vaccine. 2010;28(26):4235-4248. doi:10.1016/j. vaccine.2010.04.052

10. Brunson EK. The impact of social networks on parents' vaccination decisions. Pediatrics. 2013;131(5):e1397-1404. doi:10.1542/peds.20122452

11. Larson HJ, Jarrett C, Schulz WS, et al. Measuring vaccine hesitancy: the development of a survey tool. Vaccine. 2015;33(34):4165-4175. doi:10.1016/j.vaccine.2015.04.037

12. Luthy KE, Beckstrand RL, Callister LC. Parental hesitation in immunizing children in Utah. Public Health Nurs. 2010;27(1):25-31. doi:10.1111/phn.2010.27.issue-1 
13. Butler R, MacDonald NE, Hesitancy SWGoV. Diagnosing the determinants of vaccine hesitancy in specific subgroups: the guide to Tailoring Immunization Programmes (TIP). Vaccine. 2015;33 (34):4176-4179. doi:10.1016/j.vaccine.2015.04.038

14. Glanz JM, Wagner NM, Narwaney KJ, et al. A mixed methods study of parental vaccine decision making and parent-provider trust. Acad Pediatr. 2013;13(5):481-488. doi:10.1016/j.acap.2013.05.030

15. Moss JL, Reiter PL, Rimer BK, Brewer NT. Collaborative patientprovider communication and uptake of adolescent vaccines. Soc Sci Med. 2016;159:100-107. doi:10.1016/j.socscimed.2016.04.030

16. Opel DJ, Bahta L. How you start the conversation matters. Minn Med. 2014;97(5):50-51.

17. Dube E, Laberge C, Guay M, Bramadat P, Roy R, Bettinger J. Vaccine hesitancy: an overview. Hum Vaccin Immunother. 2013;9 (8):1763-1773. doi:10.4161/hv.24657

18. Gust DA, Darling N, Kennedy A, Schwartz B. Parents with doubts about vaccines: which vaccines and reasons why. Pediatrics. 2008;122(4):718-725. doi:10.1542/peds.2007-0538

19. Ames HM, Glenton C, Lewin S. Parents' and informal caregivers' views and experiences of communication about routine childhood vaccination: a synthesis of qualitative evidence. Cochrane Database Syst Rev. 2017;2:Cd011787.

20. Leask J. How do general practitioners persuade parents to vaccinate their children? A study using standardised scenarios. N S W Public Health Bull. 2009;20(7-8):119-124. doi:10.1071/NB08064

21. Berry NJ, Henry A, Danchin M, Trevena LJ, Willaby HW, Leask J. When parents won't vaccinate their children: a qualitative investigation of australian primary care providers' experiences. BMC Pediatr. 2017;17(1):19. doi:10.1186/s12887-017-0783-2

22. Folkhälsomyndigheten. Barriers-Motivating-Factors-Mmr-VaccinationCommunities-Low-Coverage-Sweden. Stockholm: The Public Health Agency;2015. 978-91-7603-451-4.
23. Association WM. WMA declaration of Helsinki-ethical principles for medical research involving human subjects; 2013. Available from: http://www.wma.net/en/30publications/10policies/b3/.

24. Rubin HJ, Rubin IS. Qualitative Interviewing: The Art of Hearing Data. 2nd ed. Thousand Oaks, CA: Sage; 2005.

25. Graneheim UH, Lundman B. Qualitative content analysis in nursing research: concepts, procedures and measures to achieve trustworthiness. Nurse Educ Today. 2004;24(2):105-112. doi:10.1016/j.nedt.2003.10.001

26. Jama A, Ali M, Lindstrand A, Butler R, Kulane A. Perspectives on the measles, mumps and rubella vaccination among Somali mothers in Stockholm. Int J Environ Res Public Health. 2018;15(11):2428. doi:10.3390/ijerph15112428

27. Downs JS, de Bruin WB, Fischhoff B. Parents' vaccination comprehension and decisions. Vaccine. 2008;26(12):1595-1607. doi:10.1016/j. vaccine.2008.01.011

28. Diekema DS. Provider dismissal of vaccine-hesitant families: misguided policy that fails to benefit children. Hum Vaccin Immunother. 2013;9(12):2661-2662. doi:10.4161/hv

29. Kumar D, Chandra R, Mathur M, Samdariya S, Kapoor N. Vaccine hesitancy: understanding better to address better. Isr J Health Policy Res. 2016;5:2. doi:10.1186/s13584-016-0062-y

30. Gatera M, Bhatt S, Ngabo F, et al. Successive introduction of four new vaccines in Rwanda: high coverage and rapid scale up of Rwanda's expanded immunization program from 2009 to 2013. Vaccine. 2016;34(29):3420-3426. doi:10.1016/j.vaccine.2015.11.076

31. Henrikson NB, Opel DJ, Grothaus L, et al. Physician communication training and parental vaccine hesitancy: a randomized trial. Pediatrics. 2015;136(1):70-79. doi:10.1542/peds.2014-3199

32. Betsch C, Bohm R, Airhihenbuwa CO, et al. Improving medical decision making and health promotion through culture-sensitive health communication: an agenda for science and practice. Med Decis Making. 2016;36(7):811-833. doi:10.1177/0272989X15600434
Pediatric Health, Medicine and Therapeutics

\section{Publish your work in this journal}

Pediatric Health, Medicine and Therapeutics is an international, peerreviewed, open access journal publishing original research, reports, editorials, reviews and commentaries. All aspects of health maintenance, preventative measures and disease treatment interventions are addressed within the journal. Practitioners from all disciplines are invited to submit their work as well as healthcare researchers and patient support groups. The manuscript management system is completely online and includes a very quick and fair peer-review system. Visit http://www.dovepress.com/testimonials.php to read real quotes from published authors. 